\title{
Interference ferromagnet/semiconductor/ferromagnet spin field-effect transistor
}

\author{
Th. Schäpers, ${ }^{1,2}$ J. Nitta, ${ }^{1}$ H. B. Heersche, ${ }^{1}$ and H. Takayanagi ${ }^{1}$ \\ ${ }^{1}$ NTT Basic Research Laboratories, 3-1 Morinosato Wakamiya, Atsugi-shi, Kanagawa-ken, 243-0198 Japan \\ ${ }^{2}$ Institut für Schichten und Grenzfä̈chen, Forschungszentrum Jülich, 52425 Jülich, Germany \\ (Received 31 January 2001; revised manuscript received 7 June 2001; published 10 September 2001)
}

\begin{abstract}
An interference ferromagnet/semiconductor/ferromagnet transistor is proposed, where the relative conductance difference between parallel and antiparallel magnetization oscillates as a function of gate voltage. The characteristics of a one-dimensional as well as a two-dimensional structure are calculated and compared. In both cases the interferences result in an enhanced spin signal. It is shown that by using the spin filtering effect of an interface barrier the signal can be further increased.
\end{abstract}

DOI: 10.1103/PhysRevB.64.125314

PACS number(s): 73.40.Sx, 72.25.-b, 73.23.-b

Semiconductor/ferromagnet structures have recently attracted considerable attention in the so-called field of "spintronics." 1,2 The major reason is that semiconductors offer the unique feature to control the carrier concentration by a gate electrode. Moreover, effects, like the Rashba spinorbit interaction, ${ }^{3,4}$ can add novel features to the device characteristics. $^{5-7}$ Spin injection from a ferromagnet (FM) into a semiconductor (SM) has been demonstrated by using optical detection methods. ${ }^{8,9}$ Regarding electrical detection of a spin-polarized current first results have been reported and discussed. ${ }^{10-16}$ For a diffusive semiconductor sandwiched between ferromagnetic electrodes Schmidt et al. ${ }^{17}$ pointed out that due to the large conductivity mismatch only a very low spin-polarization current can be expected. However, this situation is improved if a tunneling barrier is introduced at the interface. ${ }^{15,18}$

In case of high-mobility semiconductors, i.e., twodimensional electron gas structures, the conductivity of the semiconductor is considerably increased. Owing to the large elastic mean free path, the transport through the FM/SM interface can be described by a ballistic model. In this regime the relevant parameters for the spin injection into the semiconductors are the Fermi velocity mismatch and the exchange energy of the ferromagnet. Similarly to the diffusive regime an interface barrier can improve the degree of spin polarization of the injected carriers. ${ }^{19}$ For semiconductor nanostructures it is known that their large Fermi wavelength allows one to observe pronounced quantum interference effects. ${ }^{20}$ This raises the question as to whether the spin signal in a FM/SM/FM structure can further be improved by using quantum effects.

Here, we propose a spin-interference field-effect transistor based on a FM/SM/FM structure. The interference is adjusted by controlling the Fermi wavelength in the semiconductor using a gate electrode. ${ }^{21}$ It will be shown that due to spin-dependent transmission probabilities for parallel or antiparallel magnetization of the ferromagnetic electrodes the relative difference between the conductance of both modes strongly oscillates as a function of gate voltage. Under certain conditions even the sign can change. By tuning the Fermi wavelength in the semiconductor by the gate the spin signal can be improved considerably compared to the case where interference effects are neglected. Following the concept of increasing spin polarization by an interface barrier, ${ }^{15,18,19}$ the modification of the oscillation pattern is investigated by introducing a $\delta$-shaped barrier at each FM/SM interface. After discussing a one-dimensional transistor, the properties of a structure containing a twodimensional electron gas (2DEG) are investigated.

In order to calculate the conductance of a FM/SM/FM transistor, parabolic energy dispersion is assumed in the ferromagnet as well as in the semiconductor. As depicted in Fig. 1, a two-band model is applied for the ferromagnets with the majority and minority bands displaced by $\Delta_{h}=2 h_{0}{ }^{22-27}$ Here, $h_{0}$ is the exchange energy. The spin-flip length is assumed to be larger than the separation of the ferromagnetic electrodes so that spin-flip scattering can be neglected. Due to the much lower carrier concentration in the semiconductor compared to the ferromagnet, the bottom of the semiconductor conduction band is usually found at a considerably higher energy. The semiconductor layer can therefore be regarded as a potential step between the ferromagnets. By using a gate electrode the electron concentration in the semiconductor and thus the height of this potential step can be controlled. More generally, since in the semiconductor the potential is often nonconstant, i.e., if the gate length is shorter than the channel length, a series of potential steps of height $U_{j}$ can be used to describe the profile $\mathrm{e}^{28}$

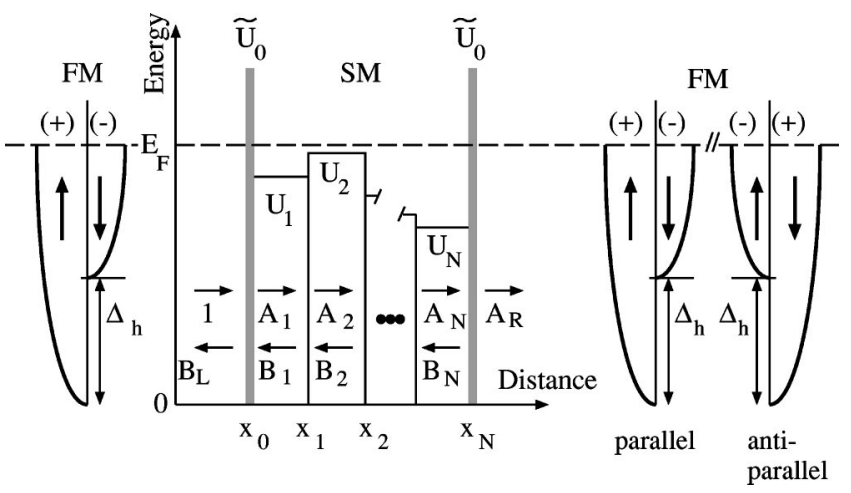

FIG. 1. Schematics of a ferromagnet/semiconductor/ ferromagnet structure (FM/SM/FM). In the ferromagnets the band of electrons with minority spins (-) is shifted by $\Delta_{h}$ with respect to the majority band $(+)$. The interface barriers are described by $\delta$-shaped potentials. The semiconductor potential profile is approximated by rectangular potential steps. 


$$
U(x)=\sum_{j=1}^{N} U_{j}\left[\Theta\left(x-x_{j-1}\right)-\Theta\left(x-x_{j}\right)\right],
$$

with $\Theta(x)$ the unit step function (see Fig. 1). In the ferromagnet, the free-electron mass $m_{e}$ and in the semiconductor and effective electron mass $m^{*}$ are assumed, respectively. Motivated by the theoretically predicted enhancement of spin injection by using interface barriers, additional tunnel barriers, e.g., oxide layers, are assumed at the FM/SM interface. In our model these barriers are described by $\delta$-shaped potentials $^{29}$

$$
\widetilde{U}(x)=\widetilde{U}_{0}\left[\delta\left(x-x_{0}\right)+\delta\left(x-x_{N}\right)\right] .
$$

Combining all contributions, the effective Hamiltonian in the free-electron approximation can be expressed as

$$
H=-\frac{\hbar^{2}}{2 m(x)} \frac{\partial^{2}}{\partial \mathbf{r}^{2}}+U(x)+\widetilde{U}(x)-h(x) \sigma .
$$

The last term represents the internal exchange energy, with $\sigma=+1$ for majority and $\sigma=-1$ for minority carriers, respectively. $h(x)=h_{0}$ in the ferromagnet, while $h(x)=0$ in the semiconductor.

The conductance of the transistor structure is obtained by calculating the transmission probability from the left to the right electrode. First, we restrict ourselves to onedimensional transport in the limit of small voltage bias. The latter implies that only electrons at the Fermi energy are regarded. A plane-wave approximation is used. In the ferromagnet material the wave functions of carriers with majority $(+)$ and minority $(-)$ spins in the left and right electrodes are

$$
\begin{aligned}
& \psi_{L, \pm}=A_{L} e^{i k_{ \pm} x}+B_{L} e^{-i k_{ \pm} x}, \\
& \psi_{R, \pm}=A_{R} e^{i k_{ \pm} x}+B_{R} e^{-i k_{ \pm} x} .
\end{aligned}
$$

Here, $k_{+}=\sqrt{2 m_{e} E_{F}} / \hbar$ and $k_{-}=\sqrt{2 m_{e}\left(E_{F}-\Delta_{h}\right)} / \hbar$ are the Fermi wave vectors of the majority $(+)$ and minority $(-)$ subbands. $E_{F}$ is the Fermi energy with respect to the bottom of the majority band. The wave function in the semiconductor can be expressed as

$$
\psi_{j}=A_{j} e^{i k_{j} x}+B_{j} e^{-i k_{j} x}, \quad 1 \leqslant j \leqslant N,
$$

with $k_{j}=\sqrt{2 m^{*}\left(E_{F}-U_{j}\right)} / \hbar$. From the boundary conditions at the interface of adjacent steps a matrix $\mathbf{M}_{j}$ can be derived, which expresses the transition from the $j$ th to the $(j+1)$ th potential step, ${ }^{28}$

$$
\left(\begin{array}{c}
A_{j+1} \\
B_{j+1}
\end{array}\right)=\mathbf{M}_{j}\left(\begin{array}{c}
A_{j} \\
B_{j}
\end{array}\right), \quad 1 \leqslant j \leqslant N-1
$$

with $\mathbf{M}_{j}$ given by

$$
\mathbf{M}_{j}=\frac{1}{2}\left(\begin{array}{cc}
\left(1+S_{j}\right) e^{-i\left(k_{j+1}-k_{j}\right) x_{j}} & \left(1-S_{j}\right) e^{-i\left(k_{j+1}+k_{j}\right) x_{j}} \\
\left(1-S_{j}\right) e^{+i\left(k_{j+1}+k_{j}\right) x_{j}} & \left(1+S_{j}\right) e^{+i\left(k_{j+1}-k_{j}\right) x_{j}}
\end{array}\right)
$$

and $S_{j}=k_{j} / k_{j+1}$. Due to the $\delta$ barrier at the FM/SM interface, the derivative of the wave function is not continuous, i.e.,

$$
\left.\frac{1}{m^{*}} \frac{d \psi_{1}}{d x}\right|_{x_{0}+}=\left.\frac{1}{m_{e}} \frac{d \psi_{L, \pm}}{d x}\right|_{x_{0}-}+\frac{2 \widetilde{U}_{0}}{\hbar^{2}} \psi_{L, \pm}\left(x_{0}\right),
$$

for the left interface. The transition from the left ferromagnet to the semiconductor can be written as

$$
\left(\begin{array}{c}
A_{1} \\
B_{1}
\end{array}\right)=\mathbf{M}_{L}^{ \pm}\left(\begin{array}{c}
A_{L} \\
B_{L}
\end{array}\right)
$$

The matrix $\mathbf{M}_{L}^{ \pm}$is given by

$$
\mathbf{M}_{L}^{ \pm}=\frac{1}{2}\left(\begin{array}{cc}
\left(1+S_{L, \pm}-2 i Z \widetilde{S}_{L, \pm}\right) e^{-i\left(k_{1}-k_{ \pm}\right) x_{0}} & \left(1-S_{L, \pm}-2 i Z \widetilde{S}_{L, \pm}\right) e^{-i\left(k_{1}+k_{ \pm}\right) x_{0}} \\
\left(1-S_{L, \pm}+2 i Z \widetilde{S}_{L, \pm}\right) e^{+i\left(k_{1}+k_{ \pm}\right) x_{0}} & \left(1+S_{L, \pm}+2 i Z \widetilde{S}_{L, \pm}\right) e^{+i\left(k_{1}-k_{ \pm}\right) x_{0}}
\end{array}\right),
$$

with $S_{L, \pm}=\left(m^{*} / m_{e}\right)\left(k_{ \pm} / k_{1}\right)$ and $\widetilde{S}_{L, \pm}=\left(m^{*} / m_{e}\right)\left(k_{+} / k_{1}\right)$. Here, the dimensionless factor $Z=m_{e} \widetilde{U}_{0} / \hbar^{2} k_{+}$was introduced, normalized to the Fermi velocity of the majority carriers in the left electrode. ${ }^{29}$ A similar matrix $\mathbf{M}_{R}^{ \pm}$, with $S_{R, \pm}=1 / S_{L, \pm}, \widetilde{S}_{R, \pm}=k_{+} / k_{ \pm}$, and the corresponding phase factors, can be obtained for the transition from the semiconductor to the majority $(+)$ and minority $(-)$ band of the right electrode.

The total transmission amplitude $A_{R}$ from the left to the right ferromagnet is obtained from the matrix $\mathbf{M}^{\alpha \beta}$,

$$
\left(\begin{array}{c}
A_{R} \\
B_{R}
\end{array}\right)=\mathbf{M}^{\alpha \beta}\left(\begin{array}{c}
A_{L} \\
B_{L}
\end{array}\right), \quad \alpha, \beta=+,-,
$$

with

$$
\mathbf{M}^{\alpha \beta}=\left(\begin{array}{cc}
M_{11}^{\alpha \beta} & M_{12}^{\alpha \beta} \\
M_{21}^{\alpha \beta} & M_{22}^{\alpha \beta}
\end{array}\right)=\mathbf{M}_{R}^{\beta}\left(\prod_{j=1}^{N-1} \mathbf{M}_{j}\right) \mathbf{M}_{L}^{\alpha},
$$

and setting $A_{L}=1$ and $B_{R}=0$. The amplitude $A_{R}$ is connected to the transmission probability by ${ }^{28}$

$$
T_{\alpha \beta}=\frac{k_{R, \beta}}{k_{L, \alpha}}\left|A_{R}\right|^{2}=\frac{k_{L, \alpha}}{k_{R, \beta}} \frac{1}{\left|M_{22}^{\alpha \beta}\right|^{2}} .
$$

In the case of parallel magnetization of both ferromagnet electrodes, the one-dimensional total conductance is given 


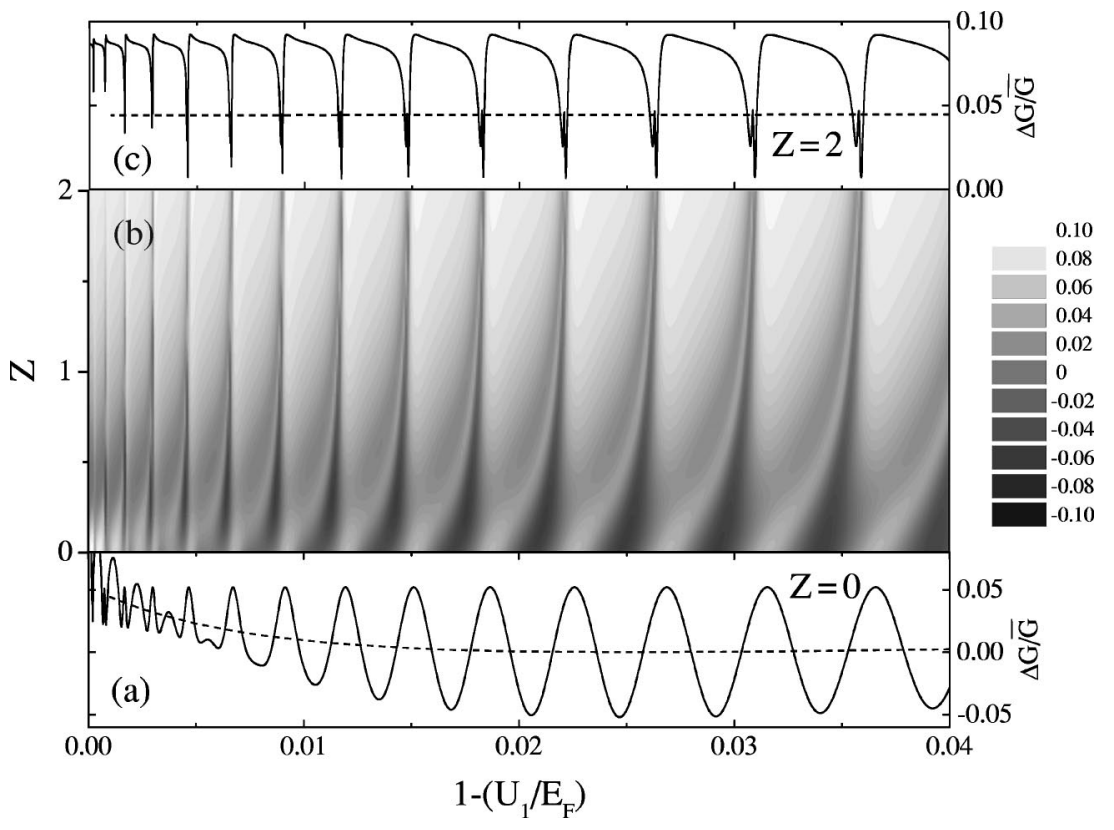

FIG. 2. Relative conductance difference $\Delta G / \bar{G}$ as a function of the normalized Fermi energy 1 $-U_{1} / E_{F}$ in the semiconductor for $\Delta_{h}=0.6 E_{F}$. (a) and (c) correspond to an interface barrier with $Z=0$ and 2, respectively. (b) shows $\Delta G / \bar{G}$ with increasing interface barrier height $Z$. The length of the semiconductor layer was assumed to be $1150 / k_{+}$corresponding to $100 \mathrm{~nm}$ at $E_{F}$ $=5 \mathrm{eV}$. The dashed lines in (a) and (c) refer to the case when interferences are neglected. by the transmission from the majority and minority bands into the corresponding majority and minority bands on the opposite side:

$$
G_{p}=\frac{e^{2}}{h}\left(T_{++}+T_{--}\right) .
$$

For the antiparallel case the conductance is given by

$$
G_{a}=\frac{2 e^{2}}{h} T_{+-} .
$$

Let us first consider a one-dimensional transistor structure, with a semiconductor quantum wire placed between two ferromagnets. Only a single subband is considered in the wire, with a constant bottom of the subband given by a single potential step $U_{1}$. The height $U_{1}$ is controllable by a gate. Consequently, the Fermi energy $E_{F}-U_{1}$ in the semiconductor and $k_{1}$ are also altered by the gate bias. In Fig. 2 the relative difference of the conductance $\Delta G / \bar{G}$, with $\Delta G$ $=\left(G_{p}-G_{a}\right)$ and $\bar{G}=\left(G_{p}+G_{a}\right) / 2$, is plotted as a function of the normalized energy in the semiconductor $1-U_{1} / E_{F}$. A typical effective electron mass of $m^{*}=0.04 m_{e}$ of an InAsbased material was taken for the semiconductor. ${ }^{30,31}$ The exchange energy was assumed to be $\Delta_{h}=0.6 E_{F}$. The length $x_{1}$ of the semiconductor layer corresponds to $100 \mathrm{~nm}$ if $E_{F}$ $=5 \mathrm{eV}$ as a typical value for $d$-band ferromagnets is assumed. $^{7}$

For no interface barriers $(Z=0)$ the relative conductance difference $\Delta G / \bar{G}$ strongly oscillates if the Fermi energy of the semiconductor is changed [Fig. 2(a)]. ${ }^{32}$ The maxima in $\Delta G / \bar{G}$ appear at energies $E_{F}-U_{1}$, where $k_{1}$ matches to $\pi n / x_{1}$, with $n=1,2,3, \ldots$ Here, the transmission probabilities $T_{++}$and $T_{--}$for parallel magnetization are equal to 1 , whereas $T_{+-}=4 r /(r+1)^{2}$, with $r=k_{-} / k_{+}=\sqrt{1-\Delta_{h} / E_{F}}$ the ratio of the wave vectors of minority and majority carriers. Since all transmission probabilities are independent of $k_{1}$, the maxima, given by $\Delta G / \bar{G}=2(r-1)^{2} /\left(r^{2}+6 r+1\right)$, only depend on $r$. The oscillations are most pronounced if the Fermi velocity $v_{1}=\hbar k_{1} / m^{*}$ of the semiconductor is adjusted to a value in between the Fermi velocities of majority and minority carriers, $v_{ \pm}=\hbar k_{ \pm} / m_{e}$, of the ferromagnet. An absolute minimum of, with the same magnitude as, the maxima is obtained if $v_{1}^{2}=v_{+} v_{-}$and if $x_{1}$ matches $2 k_{1} x_{1}$ $=(2 n+1) \pi$ with $n$ out of $1,2,3, \ldots$ at the same time. ${ }^{33}$ By comparing these results to the case where interferences are neglected [Fig. 2(a), dashed line] it is obvious that $\Delta G / \bar{G}$ can be improved largely by adjusting the interference by the gate. For increasing $Z$ values the minima in $\Delta G / \bar{G}$ shift towards higher energies $E_{F}-U_{1}$, while their widths decrease at the same time [Fig. 2(b) and 2(c)]. At $Z=2$, the average value of $\Delta G / \bar{G}$ is found to be of the order of $8 \%$, which is considerably higher than for the case without interferences [Fig. 2(c), dashed line]. The increase with $Z$ can be attributed to improved spin polarization and thus improved spin filtering due to the interface barriers. ${ }^{15,18,19}$

The characteristics of a transistor with a two-dimensional electron gas as the semiconductor are depicted in Fig. 3. For the calculation of the conductance, momentum conservation parallel to the interface was assumed and an integration over all angles of incidence was performed. In a $2 \mathrm{DEG}$ with a single subband occupied the electron concentration $n_{2 D}$ is proportional to the Fermi energy $E_{F}-U_{1}=\pi \hbar^{2} n_{2 D} / m^{*}$. Referring to $E_{F}$ and $m^{*}$, as given above, $1-U_{1} / E_{F}=0.04$ corresponds to $n_{2 D} \approx 3 \times 10^{12} \mathrm{~cm}^{-2}$, which is a reasonable value for a $2 \mathrm{DEG}$ in a semiconductor heterostructure.

For a situation, with no $\delta$-shaped interface barriers [Fig. $3(\mathrm{a})]$, the oscillations found previously for the onedimensional case are preserved. However, due to the averaging over the angles of incidence, the oscillation amplitude is lowered. Values of $\Delta G / \bar{G}$ below zero are still found, but their magnitude is considerably smaller than the corresponding adjacent maxima. As expected, $\Delta G / \bar{G}$ is decreased if $\Delta_{h}$ 


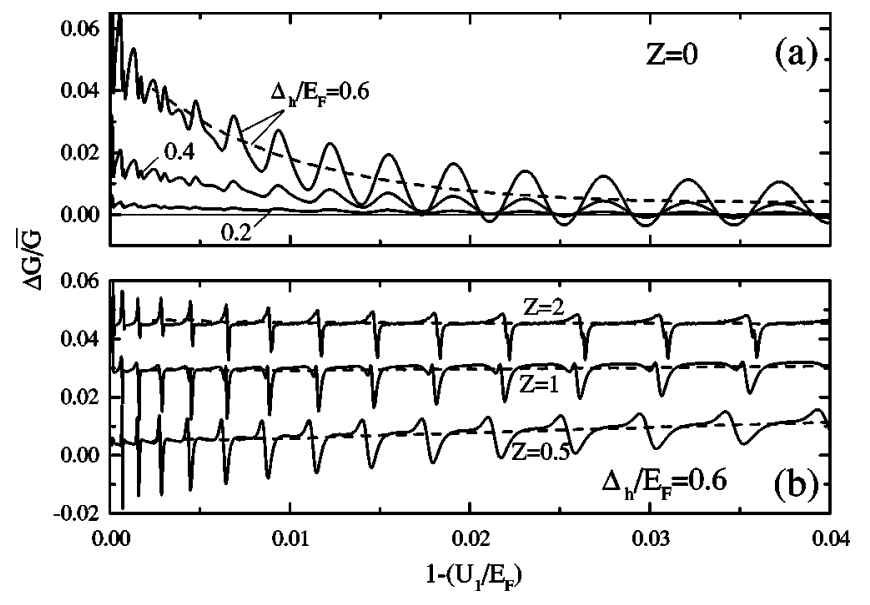

FIG. 3. $\Delta G / \bar{G}$ of a $2 \mathrm{DEG}$ as a function of normalized Fermi energy $1-U_{1} / E_{F}$ of the semiconductor. (a) shows $\Delta G / \bar{G}$ for $Z$ $=0$ with $\Delta_{h} / E_{F}$ varied as a parameter. In (b) results are plotted for $Z=0.5,1.0$, and 2.0 at $\Delta_{h} / E_{F}=0.6$. The channel length corresponds to $100 \mathrm{~nm}$ for $E_{F}=5 \mathrm{eV}$. The dashed lines refer to the case when interferences are neglected.

decreases. Because of the improved spin polarization by an interface barrier, $\Delta G / \bar{G}$ on average increases for $Z$ exceeding a value of about 1 . In contrast to the case for $Z=0$, $\Delta G / \bar{G}$ can only slightly be improved for larger $Z$ values compared to a situation where interferences are neglected (Fig. 3, dashed lines).

In Fig. 4 the characteristics of a two-dimensional transistor structure are shown, where the gate covers only part of the channel. In this geometry it is possible to improve the gate response by reducing the distance to the conducting channel. Since here the oscillations are mainly determined by the gate length, the electrode separation was increased to a value corresponding to $300 \mathrm{~nm}$. As shown in the schematics in Fig. 4, the potential of the semiconductor is approximated by three steps of same width. Only the height of the center step is controlled by the gate, while the outer ones are kept at $0.98 E_{F}$. Similar to the results show in Fig. 2, pronounced oscillations are found in $\Delta G / \bar{G}$. Due to the smaller gate length compared to the ferromagnet separation, the wavelength of the oscillation is relatively large. For $\Delta_{h} / E_{F}=0.6$ and 0.4 the oscillation strength is of the same order of magnitude as for the two-dimensional structure discussed above. In case of $\Delta_{h} / E_{F}=0.8$ the maxima in $\Delta G / \bar{G}$ exceed $4 \%$ if the carrier concentration is reduced to approximately $1 / 3$ by the gate.

Comparing the results of the transistor structure based on a semiconductor quantum wire to the structure containing a 2DEG it can be stated that in both cases an increase of the spin signal due to interference effects can be expected.

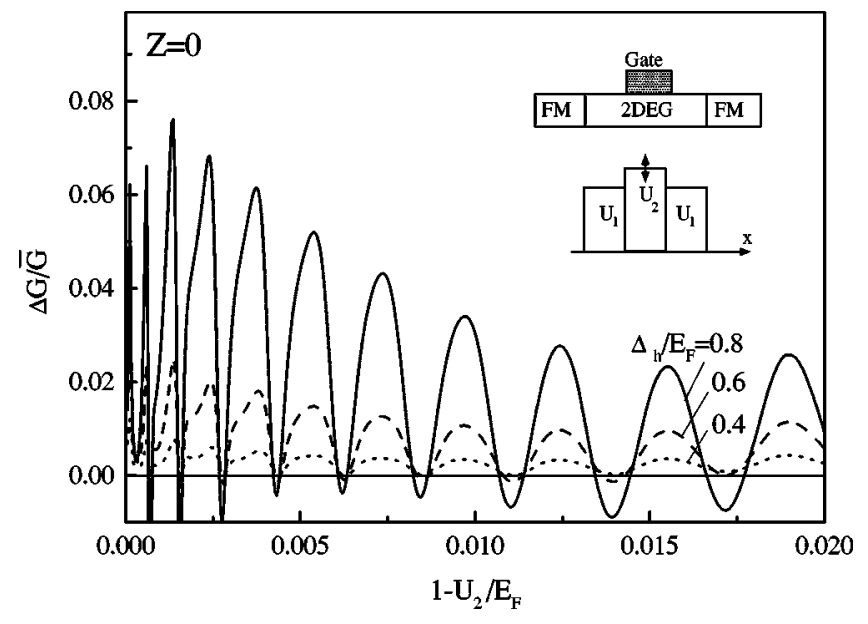

FIG. 4. $\Delta G / \bar{G}$ of a two-dimensional transistor structure as a function of the Fermi energy below the gate electrode for various exchange energies for $Z=0$. The inset shows the device structure.

However, because of averaging effects, the gain in $\Delta G / \bar{G}$ is lower for the two-dimensional structure. In order to experimentally observe the predicted oscillations clearly, a onedimensional structure should be preferred. By introducing an interface barrier, the magnitude of $\Delta G / \bar{G}$ can further be improved, also if compared to the nonresonant case. Although the average value of $\Delta G / \bar{G}$ is increased, the oscillation amplitude of $\Delta G / \bar{G}$ remains about the same if compared to the $Z=0$ case. Above that, the potential interval where $\Delta G / \bar{G}$ is lowered is decreased due to the transition from sinelike oscillations $(Z=0)$ to sharp spikes in the characteristics. Concerning a proper experimental detection of the variations in $\Delta G / \bar{G}$, this might limit the maximum height of the interface barrier which can be used.

The property that the spin-dependent conduction oscillates by varying a gate voltage is similar to the signal expected for the spin transistor proposed by Datta and Das. ${ }^{5}$ In their case the oscillations originate from the rotation of the spin orientation by the Rashba effect. ${ }^{3,4}$ An oscillating spin signal due to resonances was also predicted by Zheng et al. ${ }^{27}$ for a $\mathrm{FM} /$ normal-metal/FM structure with $\delta$-shaped interface barriers. In their case, the oscillations are studied as a function of the normal layer thickness.

In summary, we proposed an interference FM/SM/FM transistor, where the relative conductance difference between parallel and antiparallel magnetization $\Delta G / \bar{G}$ oscillates as a function of gate voltage. If the Fermi velocity in the semiconductor is adjusted close to the Fermi velocities of the ferromagnet, $\Delta G / \bar{G}$ can even change sign. The interference effects can be used to enhance $\Delta G / \bar{G}$ in a FM/SM/FM structure.

The authors would like to thank T. Koga for valuable discussions. This work was supported by the NEDO International Joint Research Program. 
${ }^{1}$ J. de Boeck, Science 357, 357 (1998).

${ }^{2}$ P. Ball, Nature (London) 404, 918 (2000).

${ }^{3}$ E.I. Rashba, Sov. Phys. Solid State 2, 1190 (1960).

${ }^{4}$ Y.A. Bychkov and E.J. Rashba, J. Phys. C 17, 6039 (1984).

${ }^{5}$ S. Datta and B. Das, Appl. Phys. Lett. 56, 665 (1990).

${ }^{6}$ H.X. Tang, F.G. Monzon, R. Lifshitz, M.C. Cross, and M.L. Roukes, Phys. Rev. B 61, 4437 (2000).

${ }^{7}$ D. Grundler, Phys. Rev. Lett. 86, 1058 (2001).

${ }^{8}$ R. Fiederling, M. Keim, G. Reuscher, W. Ossau, G. Schmidt, A. Waag, and L.W. Molenkamp, Nature (London) 402, 787 (1999).

${ }^{9}$ Y. Ohno, D.K. Young, B. Beschoten, F. Matsukara, H. Ohno, and D.D. Awschalom, Nature (London) 402, 790 (1999).

${ }^{10}$ P.R. Hammar, B.R. Bennett, M.J. Yang, and M. Johnson, Phys. Rev. Lett. 83, 203 (1999).

${ }^{11}$ S. Gardelis, C.G. Smith, C.H.W. Barnes, E.H. Linfield, and D.A. Ritchie, Phys. Rev. B 60, 7764 (1999).

${ }^{12}$ F.G. Monzon, H.X. Tang, and M.L. Roukes, Phys. Rev. Lett. 84, 5022 (2000).

${ }^{13}$ B.J. van Wees, Phys. Rev. Lett. 84, 5023 (2000).

${ }^{14}$ P.R. Hammar, M.J. Yang, and M. Johnson, Phys. Rev. Lett. 84, 5024 (2000).

${ }^{15}$ A.T. Filip, B.H. Hoving, F.J. Jedema, B.J. van Wees, B. Dutta, and S. Borghs, Phys. Rev. B 62, 9996 (2000).

${ }^{16}$ C.-M. Hu, J. Nitta, A. Jensen, J.B. Hansen, and H. Takayanagi, Phys. Rev. B 63, 125333 (2001).

${ }^{17}$ G. Schmidt, D. Ferrand, L.W. Molenkamp, A.T. Filip, and B.J. van Wees, Phys. Rev. B 62, R4790 (2000).

${ }^{18}$ E.I. Rashba, Phys. Rev. B 62, 16267 (2000).

${ }^{19}$ H.B. Heersche, Th. Schäpers, J. Nitta, and H. Takayanagi,
Phys. Rev. B (to be published).

${ }^{20}$ C.W.J. Beenakker and H. van Houten, in Solid State Physics, edited by H. Ehrenreich and D. Turnbull (Academic, New York, 1991), Vol. 44, p. 1

${ }^{21}$ Interferences in metal/semiconductor/metal structures have been demonstrated before by using a superconductor as a metal; see, e.g., H. Takayanagi, T. Akazaki, and J. Nitta, Phys. Rev. B 51, 1374 (1995).

${ }^{22}$ J.C. Slonczewski, Phys. Rev. B 39, 6995 (1989).

${ }^{23}$ G.E.W. Bauer, Phys. Rev. Lett. 69, 1676 (1992).

${ }^{24}$ M.J.M. de Jong and C.W.J. Beenakker, Phys. Rev. Lett. 74, 1657 (1995).

${ }^{25}$ X. Zhang, B.-Z. Li, G. Sun, and F.-C. Pu, Phys. Rev. B 56, 5484 (1997).

${ }^{26}$ Y. Qi, D.Y. Xing, and J. Dong, Phys. Rev. B 58, 2783 (1998).

${ }^{27}$ Z. Zheng, Y. Qi, D.Y. Xing, and J. Dong, Phys. Rev. B 59, 14505 (1999).

${ }^{28}$ Y. Ando and T. Itoh, J. Appl. Phys. 61, 1487 (1987).

${ }^{29}$ G.E. Blonder, M. Tinkham, and T.M. Klapwijk, Phys. Rev. B 25, 4515 (1982).

${ }^{30}$ J. Nitta, T. Akazaki, H. Takayanagi, and T. Enoki, Phys. Rev. Lett. 78, 1335 (1997).

${ }^{31}$ Th. Schäpers, G. Engels, J. Lange, Th. Klocke, M. Hollfelder, and H. Lüth, J. Appl. Phys. 83, 4324 (1998).

${ }^{32}$ A change of $\Delta G / G$ as a function of the normal metal layer thickness in a FM/insulator/normal-metal/FM structure was reported by J.S. Moodera et al., Phys. Rev. Lett. 83, 3029 (1999).

${ }^{33}$ The parameter set used for the calculation shown in Fig. 2, $x_{1}$, does not comply with this condition. 